\title{
Bitcoin: a moeda do ciberespaço
}

\author{
Hindenburgo Francisco Pires \\ IGEOG/UERJ
}

\section{revista}

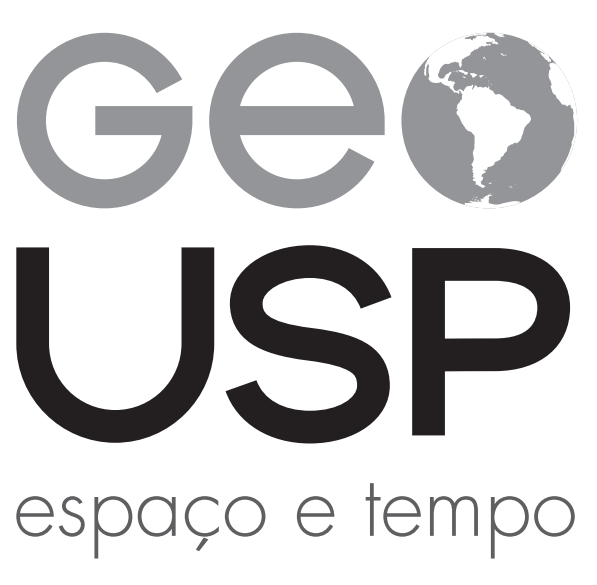

Volume $21 \cdot n^{\circ} 2(2017)$

Como citar este artigo:

PIRES, H. F. Bitcoin: a moeda do ciberespaço. Geousp Espaço e Tempo (Online), v. 21, n. 2, p. 407-424, agosto. 2017. ISSN 2179-0892.

Disponível em: < http://www.revistas.usp.br/geousp/ article/view/134538> doi: 10.11606/issn.2179-0892. geousp.2017.134538.

\section{(c) $\underset{\mathrm{BY}}{(1)}$ (8)}

Este artigo está licenciado sob a Creative Commons Attribution 4.0 License. 


\section{Bitcoin: a moeda do ciberespaço}

\section{Resumo}

A recente expansão global do uso de moedas virtuais passou a ser objeto de debate nos três últimos Fóruns das Nações Unidas sobre Governança da Internet (IGF), de 2014, 2015 e 2016. A capitalização e a rápida expansão geográfica do uso de criptomoedas chamaram a atenção de pesquisadores e mobilizaram representantes da sociedade civil e empresários em várias partes do mundo, que demonstraram interesse no desenvolvimento de inovações impulsionadas pela expansão do uso globalizado desse novo tipo de atividade econômica. Os principais objetivos deste trabalho são: analisar como a recente crise financeira do capitalismo influiu no surgimento e na expansão geográfica do uso de criptomoedas; refletir sobre serviços e atividades que usam criptomoedas, bitcoin e blockchain; e debater os desafios colocados pelo crescimento desse uso.

Palavras-chave: Desregulação financeira. Governança da internet. Moedas virtuais. Blockchain. Bitcoins.

\section{Bitcoin: the currency of cyberspace}

\section{Abstract}

The recent global expansion of the use of virtual currency became an object of debate at the last three United Nations Internet Governance Forums (IGF) for 2014, 2015 and 2016. Capitalization, rapid and geographic expansion of the use of cryptocurrencies drew attention to researchers, mobilized representatives of civil society, and entrepreneurs in various parts of the world, which showed interest on the development of innovations driven by the expansion of globalized use of this new type of economic activity. This work has as main objectives: to analyze how the recent financial crisis of capitalism influenced the emergence and the geographical expansion of the uses of cryptocurrencies; reflect on services and activities that use cryptocurrencies, bitcoins and blockchains, and discuss the challenges posed by the growth of the uses of cryptocurrencies.

Keywords: Financial Deregulation. Internet Governance. Virtual Currency. Blockchain. Bitcoins. 


\section{Introdução}

A expansão global recente do uso de moedas virtuais passou a ser objeto de debate nos três últimos Fóruns das Nações Unidas sobre Governança da Internet (IGF), de 2014, 2015 e 2016.

No IGF de 2014, em Istambul, na Turquia, o debate centrou-se em questões relacionadas com ao desenvolvimento de mecanismos de tributação e controle técnico dos meios de pagamentos feitos com moedas virtuais. ${ }^{1} \bigcirc$ debate multissetorial envolvendo pesquisadores, representantes da sociedade civil e empresários, orientou-se na elaboração de leis para garantir a proteção da privacidade, o anonimato e a luta contra o financiamento internacional de atos criminosos a nível mundial.

No IGF de 2015, em João Pessoa, esse debate multissetorial centrou-se nas inovações introduzidas pela bitcoin ${ }^{2}$ e pela nova plataforma ethereum e no protocolo criptografado, baseado numa cadeia de blocos (blockchains) para mineração de criptomoedas, mais confiável para a internet. Esse uso de tecnologias baseadas em cadeias de blocos é um desafio assumido por várias e importantes corporações internacionais. ${ }^{3}$

No IGF de 2016, em Jalisco, no México, a Internet Society (ISOC) e outros segmentos multissetoriais tentaram formalizar um debate para a elaboração de propostas de consolidação de instrumentos legais que garantam a regulamentação financeira do uso de tecnologias baseadas em blockchain, no mercado global. ${ }^{4}$

A capitalização, a expansão geográfica rápida e cumulativa do uso de moedas virtuais chamou a atenção e mobilizou organizações da sociedade civil e empresários, que demonstraram interesse sobre o desenvolvimento de inovações desse novo tipo de atividade.

Diante deste contexto, este trabalho tem os seguintes objetivos: (a) analisar como a desregulação financeira produzida pela crise de 2007-2008 influiu no surgimento do projeto de desenvolvimento global de moedas virtuais; (b) debater a importância da inovação tecnológica introduzida pela blockchain; (c) revelar como ocorre a expansão geográfica de novos mercados e serviços financeiros, que utilizam moedas virtuais; (d) refletir sobre a formação de uma nova geração de serviços financeiros globais, principalmente em atividades vinculadas à nova economia digital; e (e) debater os desafios colocados pelo crescimento dos usos de moedas virtuais no ciberespaço.

\section{Aspectos metodológicos da pesquisa}

Este artigo é resultado de minhas reflexões sobre temas que envolvem conteúdos de inovações tecnológicas e suas relações com a geografia do ciberespaço e das finanças.

\footnotetext{
$1 \bigcirc$ conceito de criptomoeda, ou moeda virtual, desenvolvido neste trabalho é baseado no relatório Application of FinCEN's Regulations to Virtual Currency Mining Operations, elaborado pela Rede de Execução de Crimes Financeiros, ou Financial Crimes Enforcement Network (FinCEN), de 30 de janeiro de 2014, segundo o qual: "Moeda 'virtual' é um meio de troca que opera como uma moeda em alguns ambientes, mas não tem todos os atributos da moeda real. Em particular, a moeda virtual não tem status de curso legal em qualquer jurisdição. A orientação aborda a moeda virtual 'conversível'. Esse tipo de moeda virtual, ou tem um valor equivalente em moeda real, ou atua como um substituto para a moeda real" (Fincen, 2014).

2 Segundo o repositório de software Sourceforge ([s.d.]): "A Bitcoin é um sistema descentralizado de dinheiro eletrônico Peer-to-Peer (P2P) sem um servidor central ou partes confiáveis. Os usuários têm as chaves criptográficas para o seu próprio dinheiro e transacionam diretamente na rede com outros usuários do sistema".

3 Ver vídeos do IGF 2015 de João Pessoa-BR (Sched, 2015) e também IGF (2015).

4 Ver vídeo e documento do IGF 2016 de Jalisco-MX, na sessão Blockchain Technologies (IGF, 2016).
} 
Os primeiros levantamentos bibliográficos efetuados por mim sobre a temática das moedas virtuais começaram a ser efetuados a partir de estudos e trabalhos apresentados em eventos, sobre fetichismo da forma dinheiro, o dinheiro no ciberespaço e as crises do capitalismo em $2012 .{ }^{5}$

$\bigcirc$ fato também de ter assistido alguns debates sobre a temática das criptomoedas, no Fórum das Nações Unidas sobre Governança da Internet (IGF) de 2015, realizado em João Pessoa, também serviu de estímulo para despertar o interesse sobre o conteúdo dessa complexa temática.

As fontes de informações cruciais para o desenvolvimento desse trabalho foram as seguintes: FiatLeak, Map of Coins, CoinGecko, CoinCap, Sourceforge, GitHub e CoinMap.

a) $\bigcirc$ site Fiatleak fornece informações geográficas, em tempo real na internet, sobre a circulação diária e o fluxo mundial de moedas para BTC. As informações fornecidas pelo site Fiatleak oferece a possibilidade de se estabelecer uma estimativa temporal sobre o fluxo mundial de moedas para BTC no ciberespaço (Figura 1);

\section{Figura 1 - Fluxo mundial de bitcoins (BTC) em 2017}
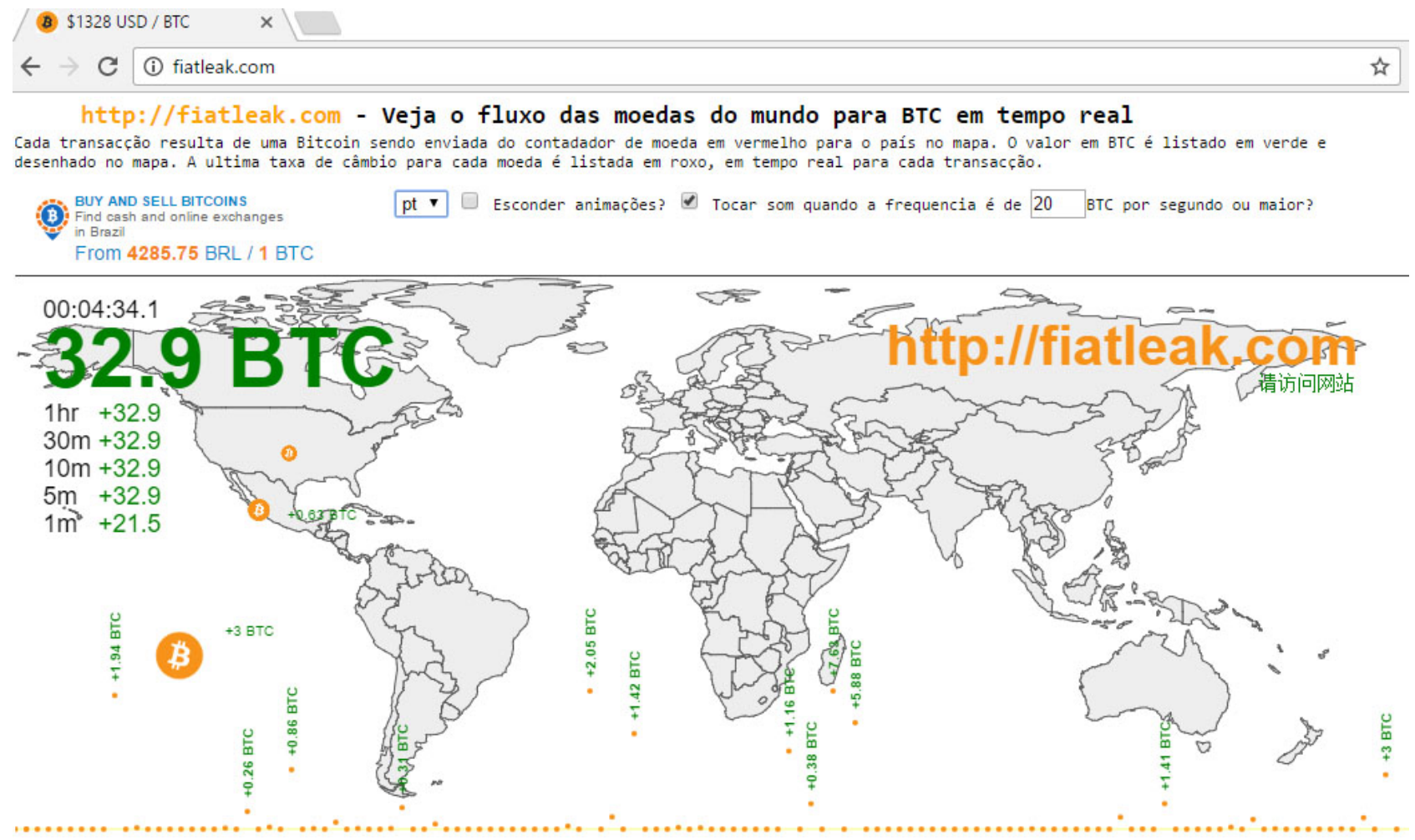

fonte: FiatLeak, em 2017.

b) $\bigcirc$ site Map of Coins é uma importante ferramenta para quem deseja explorar e conhecer a história das criptomoedas. Esse site permite a ver a disseminação histórica da utilização dos algoritmos de hash de quatro grupos de moedas virtuais: Bitcoin (BTC), Bytecoin (BCN), NXT technology (NXT) e Ripple (XRP). ○ Map of Coins fornece também informações detalhadas dos projetos de desenvolvimento tecnológico de softwares Peer-to-Peer (P2P) de oito grandes grupos de moedas virtuais: Bitcoin, Ripple, CryptoNote, NXT technology, Ethereum, XCurrency, Bitshares e o ZeroCash;

5 Sobre este tópico, ler meu artigo Capitalismo gestor de dinheiro e desregulação financeira: o endividamento dos EUA e das economias centrais (Pires, 2012). 
c) $\bigcirc$ site CoinGecko traz informações e estatísticas econômicas de 423 moedas virtuais, sobre uma ampla gama de conteúdos como: (a) mercado de capitalização (MktCap), (b) liquidez, (c) desenvolvedor, (d) comunidade (redes sociais), e (e) de interesse público. Os conteúdos abordados neste trabalho foram os itens: (a) mercado de capitalização e (d) comunidade (redes sociais) (Figura 2);

Figura 2 - Imagem da tela do site CoinGecko

\begin{tabular}{|c|c|c|c|c|c|c|c|c|c|c|c|c|c|c|}
\hline \multicolumn{15}{|c|}{ tps://www.coingecko.com/pt } \\
\hline & -3) $\mathrm{Co}$ & inGecko & & & Grá & ico & Mercadori & $\mathrm{s}$ Beta & $4 \mathrm{BC}$ & z $\quad \cdots$ & & ogar & strar & - Português - \\
\hline \multicolumn{3}{|c|}{$\begin{array}{l}\text { B-BTC- } \\
\text { Todos os algoritmos de hash - }\end{array}$} & \multirow{2}{*}{$\begin{array}{l}\mathbf{O} \\
\text { Total IE } \\
\quad \%\end{array}$} & \multirow{2}{*}{ 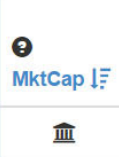 } & \multirow{2}{*}{$\begin{array}{l}\mathbf{0} \\
\text { Liquidez } ! \bar{\Xi} \\
\quad \rightleftarrows\end{array}$} & \multicolumn{2}{|c|}{$\begin{array}{l}\boldsymbol{\Theta} \\
\text { Desenvolvedor } 1\end{array}$} & \multicolumn{3}{|c|}{$\begin{array}{l}\boldsymbol{\theta} \\
\text { Comunidade } \downarrow \equiv\end{array}$} & \multicolumn{3}{|c|}{$\begin{array}{l}\mathbf{9} \\
\text { Interesse Público } \\
\downarrow \equiv\end{array}$} & \multirow{2}{*}{$\begin{array}{l}\begin{array}{l}\text { Últimos } 7 \\
\text { dias }\end{array} \\
\text { छ }\end{array}$} \\
\hline \# & Moec & $a\rfloor_{z}^{A}$ & & & & $\%$ & $\star$ & \langle\rangle & $\%$ & 0 & < & $\%$ & - & \\
\hline 1 & $\begin{array}{l}\text { (B) } \\
\text { BTC }\end{array}$ & 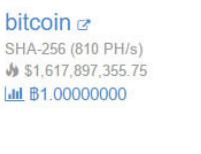 & 97 & $\begin{array}{l}\text { B16,298,437 } \\
\text { - } 0.981 \%\end{array}$ & B124143.76 & $\begin{array}{l}98 \\
w\end{array}$ & $\begin{array}{l}12252 \\
\text { p } 7973 \\
\text { @ } 1395 \\
\text { e } 3099 \\
\text { O } 2629\end{array}$ & $\begin{array}{l}m \\
4453 \\
4 \\
401 \\
401 \\
272 \\
272\end{array}$ & $\begin{array}{l}95 \\
w\end{array}$ & 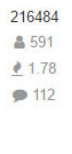 & $\begin{array}{c}\underset{f}{f} \\
30445 \\
\qquad \\
175637\end{array}$ & 84 & $\begin{array}{c}b \\
11,600,000 \\
\underset{11589}{0}\end{array}$ & $\begin{array}{l}\text { B1.00000000 } \\
\text { Comprar } \\
\text { Vender }\end{array}$ \\
\hline 2 & $\underset{\text { ETH }}{\stackrel{\Delta}{y}}$ & $\begin{array}{l}\text { ethereum } \square \\
\text { Ethash (N/A) } \\
\text { tull B0.05083615 }\end{array}$ & 86 & 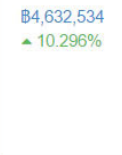 & B174799.60 & $\begin{array}{l}93 \\
\underline{w}\end{array}$ & $\begin{array}{l}3629 \\
y 1268 \\
\text { @ } 500 \\
\text { O } 2038 \\
\text { O } 1755\end{array}$ & $\begin{array}{l}\text { h } \\
804 \\
38 \\
88 \\
5 \\
89 \\
89\end{array}$ & $\begin{array}{l}75 \\
\text { 送 }\end{array}$ & $\begin{array}{l}35747 \\
\mathbf{2} 196 \\
\stackrel{2}{2.22} \\
-35.0\end{array}$ & $\underset{\substack{f \\
33423 \\
\boldsymbol{v} \\
65222}}{\underset{6}{f}}$ & 60 & $\begin{array}{c}\vec{b} \\
803,000 \\
\mathbf{0} \\
21315\end{array}$ & $\begin{array}{l}\text { B0.05083615 } \\
\text { Comprar } \\
\text { Vender }\end{array}$ \\
\hline 3 & $\begin{array}{l}\text { (1) } \\
\text { LTC }\end{array}$ & $\begin{array}{l}\text { litecoin } ₫ \\
\text { Scrypt (3.61 TH/s) } \\
\text { \$ } \$ 120.153 .164 .67 \\
\text { lall B0.01110738 }\end{array}$ & 81 & $\begin{array}{l}\text { B564,780 } \\
-4.662 \%\end{array}$ & B58319.14 & $\underset{w}{90}$ & $\begin{array}{l}1229 \\
\text { Y } 888 \\
\text { O } 255 \\
\text { O } 153 \\
\text { o } 147\end{array}$ & $\begin{array}{l}\text { m } \\
66 \\
\text { sat } \\
27 \\
\text { J } \\
59\end{array}$ & 67 & $\begin{array}{l}24067 \\
\mathbf{1} 120 \\
\stackrel{1.57}{1.57} \\
32.9\end{array}$ & 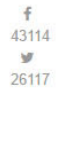 & 43 & $\begin{array}{c}b \\
726,000 \\
\mathbf{0} \\
149874\end{array}$ & $\begin{array}{l}\text { B0.01110738 } \\
\text { Comprar } \\
\text { Vender }\end{array}$ \\
\hline
\end{tabular}

fonte: CoinGecko, em 2017.

d) $\bigcirc$ site CoinCap, assim como o site CoinGecko, fornece, em tempo real um Index de 552 modalidades de criptomoedas, com informações referentes ao mercado de capitalização, preços e suprimento de criptomoedas;

e) $O$ portal Sourceforge (Pires, 2010, p. 29) é o mais importante repositório de códigos fontes do mundo para desenvolvedores de softwares open source e colaborativos. No Sourceforge, podemos ter acesso ao código fonte e softwares utilizados para a mineração de várias criptomoedas (Figura 3);

\section{Figura 3 - Imagem do projeto Bitcoin no Sourceforge}

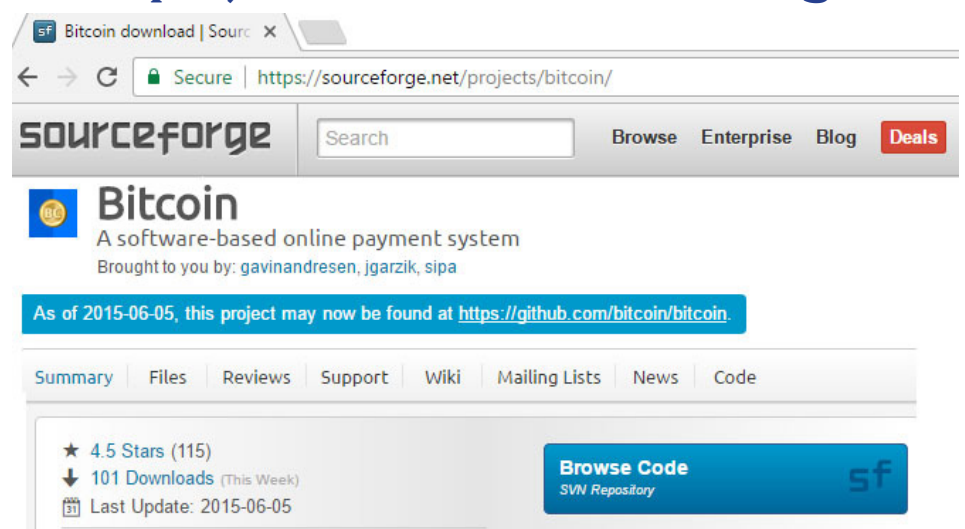

fonte: Sourceforge, em 2017. 
f) $\bigcirc$ portal de projetos GitHub é também um repositório em que é possível compartilhar com desenvolvedores conteúdos sobre projetos de desenvolvimento de softwares de moedas virtuais; e

g) $\bigcirc$ CoinMap é um guia que fornece informações e mapas, a partir do Open Street Map, sobre serviços, lojas de varejo, restaurantes e atividades comerciais que utilizam a BTC como moeda de troca. $\bigcirc$ CoinMap também permite acompanhar com mapas de "manhas de calor" a evolução histórica da instalação de atividades vinculadas ao uso de criptomoedas. Por isso, o CoinMap muitas vezes é criticado pela imprecisão de suas fontes coletadas na internet, mesmo assim é uma importante ferramenta para o estudo geográfico da evolução territorial do uso dessas moedas virtuais;

No entanto, como ocorre em qualquer pesquisa, a escolha de fontes de informação pode trazer imprecisões, e informações que podem ser contestadas, por meio de outras fontes ou bases de dados mais detalhadas, produzidas por pesquisas efetuadas no locus do objeto da pesquisa.

$\bigcirc$ desenvolvimento dos resultados dessa pesquisa foi apresentado no Seminário Internacional de Geografia e Finanças realizado em São Paulo, na Universidade de São Paulo, em abril de 2016, e também, no Annual Meeting of the American Association of Geographers, realizado na cidade de Boston, em abril de 2017 (Pires, 2017).

\section{A crise de 2007-2008 e o surgimento da inovação tecnológica da cadeia de blocos, ou blockchain: a bitcoin (BTC)}

É possível afirmar que a crise financeira internacional de 2007 e 2008, impulsionada pela globalização neoliberal e a desregulamentação financeira, conduziu à instabilidade do valor das moedas e geraram uma insegurança econômica extraordinária.

A expansão do endividamento dos EUA e das economias centrais foram reflexos deste cenário de incertezas do capitalismo (Pires, 2012). Certamente o surgimento de tecnologia baseada em algoritmos criptografados em transações financeiras, como a tecnologia da blockchain, representou uma reação inovadora à falta de limites e regras, impostas pelo cassino financeiro do capitalismo global, e também à busca de estabilidade no valor das moedas.

Criado em 2008 por Satoshi Nakamoto (Hacker?!), a BTC é uma criptomoeda concebida como uma forma criptografada do dinheiro capaz de subverter ou transgredir a regulamentação jurídica de Estados e agentes financeiros globais territorializados.

A BTC foi desenvolvida a partir de uma arquitetura de redes de computadores descentralizada (território-rede), configurada por pontos de articulação interconectados via P2P. Os registros dos dados transacionados na rede P2P são operados em uma cadeia de blocos de algoritmos, que realiza o processamento dos dados por meio de criptografia. A BTC é a 
inovação financeira mais importante do período atual. Operações com BTC requerem forte capacidade de processamento e conhecimento para operar com softwares sofisticados no mercado emergente de moedas virtuais.

É possível verificar o crescimento dos usos de moedas virtuais em várias atividades e atualmente, o valor total de BTC na cadeia de blocos é, aproximadamente, de 20 bilhões de dólares, ou 0,025\% do produto interno bruto (PIB) global, de cerca de 80 trilhões de dólares (Schwab, 2016, p. 156-157).

A circulação e o fluxo mundial de moedas para BTC podem ser acompanhados em tempo real, por meio do FiatLeak. Em poucas horas acompanhando esse fluxo no FiatLeak, pode-se observar que o volume maior de moedas do mundo mineradas virtualmente para se transformar em BTC se direciona, com mais intensidade e regularidade, para os EUA, em primeiro lugar; para a China, em segundo lugar e para a Europa, em terceiro lugar.

Em 2014, o número de pessoas que utilizavam a BTC no mundo, era de dois milhões, em um mercado de dez bilhões de dólares. Naquele momento, os professores Yelowitz e Wilson, a partir do Google Trends, ${ }^{6}$ traçaram o perfil dos usuários de BTC que também é semelhante às outras criptomoedas. Segundo eles existem quatro tipos de perfis de usuários: (a) programadores e aficionados de tecnologia; (b) investidores e especuladores de mercados financeiros; (c) antissistema ou militantes anarquistas; (d) hackers e criminosos ligados ao dinheiro de lavagem de dinheiro.

\section{A capitalização e a expansão de sistemas de gerenciamento de carteiras de moedas virtuais nas redes sociais}

uso de criptomoedas e a expansão do uso da tecnologia blockchain estão provocando uma grande transformação no mundo financeiro do século XXI. Como já observado anteriormente, a tecnologia da BTC representou uma reação "anárquica" não intencional à falta de instrumentos normativos e regulatórios para o funcionamento do sistema financeiro internacional.

$\bigcirc$ uso de novas tecnologias descentralizadas capazes de gerar criptomoedas, baseadas na blockchain, tornou-se o principal desafio assumido pelas principais corporações financeiras e comerciais do período atual, como IBM, Google, Apple, Microsoft, Samsung e Amazon.

$\bigcirc$ mercado financeiro, ao sabor de interesses de uma cleptocracia de banqueiros, representa no capitalismo uma aposta alavancada em um futuro sem controle e desregulado. A crise financeira de 2007-2008 deixou isso muito claro, por que os 10 bancos globais mais importantes do mundo gastam anualmente o equivalente a 26 trilhões de dólares anuais, que circulam sem controle.

Segundo ainda o site CoinCap, as 10 maiores moedas virtuais movimentaram no item mercado de capitalização, em 25 de abril de 2017, o equivalente a 29 bilhões de dólares (Tabela 1), nesse mercado a BTC concentrou $71,6 \%$ das operações de mineração de moedas virtuais.

6 Ver a pesquisa de Yelowitz e Wilson (2015). 


\section{Tabela 1 - Lista das dez maiores moedas virtuais no item mercado de capitalização em bilhões de dólares}

\begin{tabular}{|c|c|c|c|c|}
\hline n. & ícones & criptomoedas & $\begin{array}{l}\text { Cap. Market CoinGecko } \\
\text { (1) }\end{array}$ & $\begin{array}{l}\text { Cap. Market CoinCap } \\
\text { (2) }\end{array}$ \\
\hline 1 & & Bitcoin & $20.212 .513 .892,65$ & $20.816514 .953,00$ \\
\hline 2 & & Ethereum & $4.440 .8845 .088,07$ & $4.536 .090 .075,00$ \\
\hline 3 & & Ripple & $1.206 .596 .990,15$ & $1.224 .752 .416,00$ \\
\hline 4 & & Litecoin & $735.846 .013,58$ & $741.673 .879,00$ \\
\hline 5 & & Dash & $522.414 .574,00$ & $523.250 .780,00$ \\
\hline 6 & & $\begin{array}{l}\text { Ethereum } \\
\text { Classic }\end{array}$ & 293.721.287,09 & $358.597 .958,00$ \\
\hline 7 & & NEM & $290.365 .871,87$ & $333.137 .804,00$ \\
\hline 8 & & Monero & $288.680 .590,11$ & $281.151 .645,00$ \\
\hline 9 & & Angur & $131.010 .000,00$ & $148.030 .805,00$ \\
\hline 10 & & MaidSafeCoin & $102.100 .802,22$ & $104.544 .133,00$ \\
\hline \multicolumn{3}{|r|}{ total } & $28.224 .095 .109,74$ & $29.067 .744 .448,00$ \\
\hline
\end{tabular}

fontes e dia de obtenção dos dados: (1) CoinGecko, em 23 abr. 2017. (2) CoinCap, em 25 abr. 2017. elaboração: $\bigcirc$ autor.

Tomando como base também os dados do site CoinGecko, as dez maiores moedas virtuais, representadas segundo o item comunidade, nas redes sociais, apresentaram, em março de 2017, 1,07 milhões de usuários conectados a três redes sociais: Reddit, com 373 mil usuários; Facebook, 199 mil usuários; e Twitter, 507 mil usuários. Os usuários do Twitter ocupam um papel destaque e prevalecem como a maior comunidade (Gráfico 1).

Nas comunidades de usuários de moedas virtuais, muitas vezes, o uso estabelecido está vinculado aos seguintes nichos: econômico, identidade de gênero, identidade social e política. 


\section{Gráfico 1 - Comunidades usuárias de criptomoedas nas redes sociais}

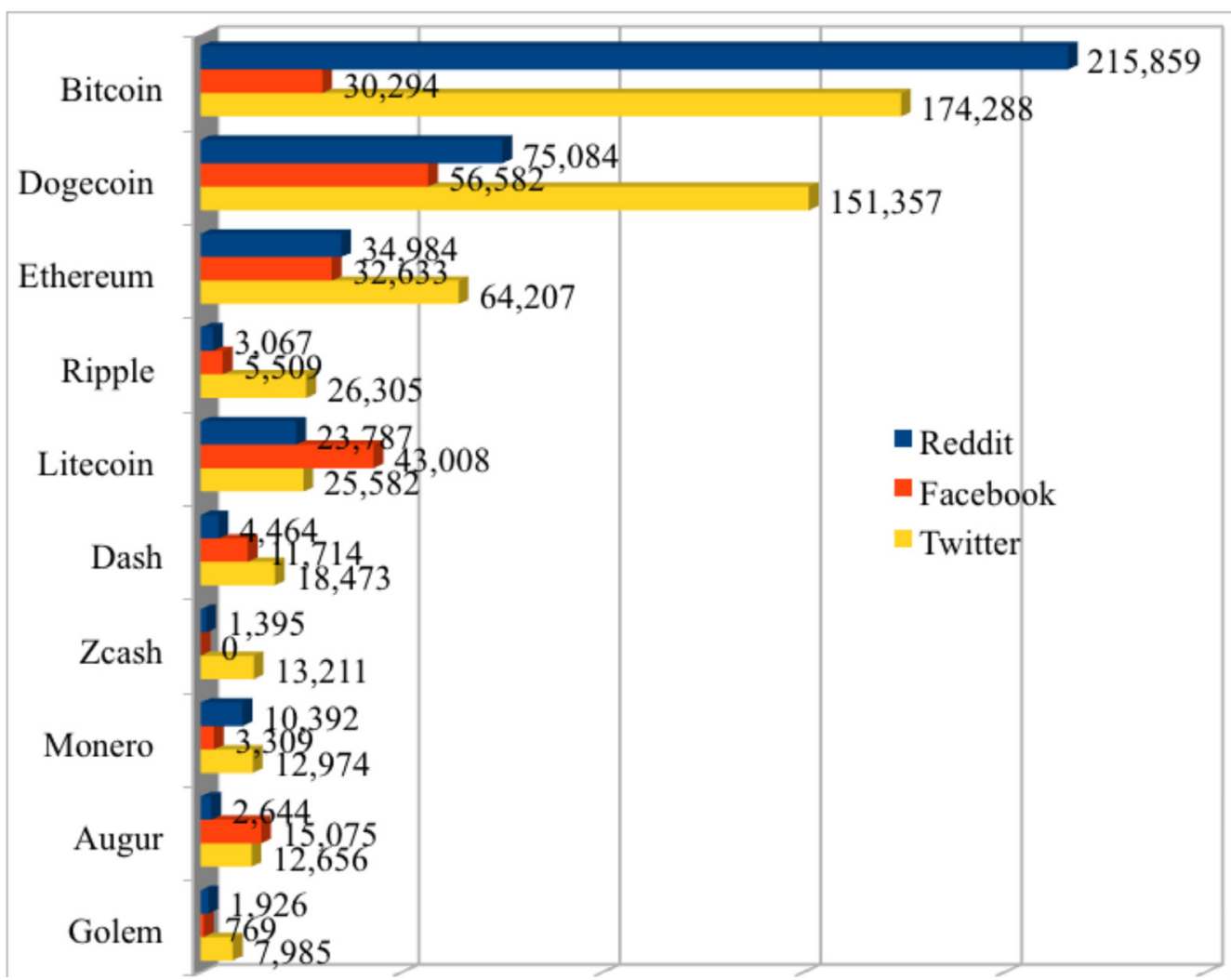

fonte: CoinGecko, em 23 abr. 2017. elaboração: $\bigcirc$ autor.

Na África do Sul, algumas comunidades utilizam moedas virtuais, como moeda social nos serviços (pagar eletricidade), nas trocas comerciais e nas operações de comércio justo.?

O crescimento anual dos sistemas de gestão de carteiras de BTC convergiu para a adoção do sistema que assumiu o pioneirismo no desenvolvimento da tecnologia de mineração de moedas virtuais, por isso, de 2008 a 2015, o número de desenvolvedores de softwares que passaram a utilizar a BTC como padrão de moeda virtual, cresceu de forma expressiva (Gráfico 2).

\section{Gráfico 2-Crescimento do número de desenvolvedores da BTC (2008-2015)}

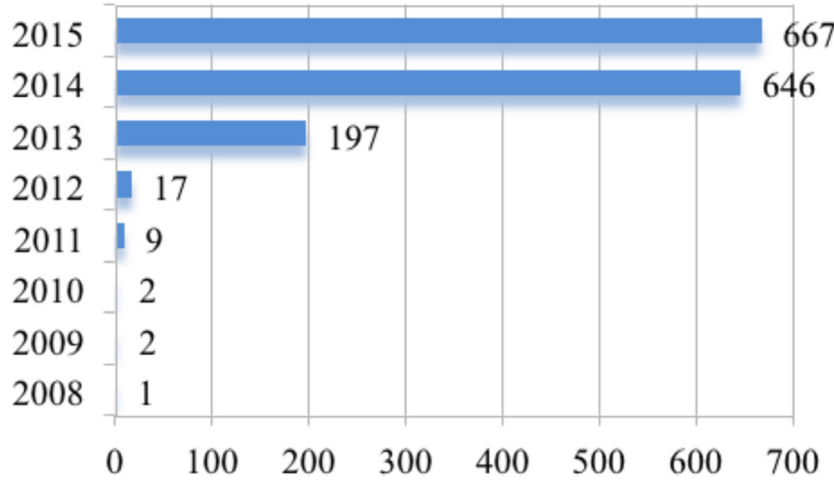

fonte: Map of Coins, em 2017. elaboração: $\bigcirc$ autor.

7 Conferir o vídeo sobre o trabalho da organização Banky Moon (2016). 


\section{Repositórios de softwares e algoritmos de hash das moedas virtuais no ci- berespaço}

Para adquirir ou minerar a BTC é preciso: em primeiro lugar, instalar o software da BTC, disponibilizado por um dos dois principais repositórios de armazenamento de softwares do mundo, os portais Sourceforge ${ }^{8}$ ou GitHub, o passo seguinte é criar uma carteira de crédito pessoal que será operada pelo software instalado ou cliente de BTC, que mediará às operações de compra na internet; em segundo lugar, o sistema cria um código/endereço/registro criptografado da operação, que é utilizado para identificar e gerar uma chave, que passa a ser a parte pública da operação, só o agente contratado tem acesso a ela; em terceiro lugar, é efetuada uma operação, que pode demorar até 10 minutos de delay, para criar função (hash) ou um algoritmo de dispersão criptográfica, ou um código/registro para validar a transação, também chamado de processo mineração de BTC; em quarto lugar, essa função hash ou algoritmo de hash (SHA256d) se junta a uma cadeia de blocos de operações realizadas com BTC. E assim se começa a operar com bitcoins.

O portador da BTC é uma pessoa anônima, que concorda participar de um processo consensuado de aceitação das regras de uso por todos da tecnologia blockchain.

Os algoritmos de hash desempenham um papel central na mineração de cripromoedas. O primeiro algoritmo criado para produzir a BTC foi concebido para gerar matematicamente o número fixo de 21 milhões de bitcoins (Sayad, 2015, p. 18).

$\mathrm{Na}$ lista das maiores moedas virtuais, existe nove importantes algoritmos de hash9 muito usados (Quadro 1).

\section{Quadro 1 - Criptomoedas e algoritmos de hash utilizados desde 2009}

\begin{tabular}{|c|c|c|c|c|c|}
\hline ícones & criptomoedas & siglas & A.Hash & fundadores & ano \\
\hline (B) & Bitcoin & BTC & Sha-256d & Satoshi Nakamoto & 2009 \\
\hline 趛 & Litecoin & LTC & ScryptN & Charles Lee & 2011 \\
\hline$\infty$ & NXT & NXT & Proof-of-Stake & Dave Pearce e Travin Keith & 2013 \\
\hline-8 & Ripple & XRP & ECDSA & Chris Larsen e Jed McCaleb & 2013 \\
\hline$M$ & Monero & XMR & Cryptonigh & Moreno Core team & 2014 \\
\hline$\Xi$ & Dash & DASH & $\mathrm{X} 11$ & Evan Duffield e Kyle Hagan & 2014 \\
\hline$\theta$ & Ethereum & ETH & Ethash-Dagger Hashimoto & Vitalik Buterin & 2015 \\
\hline 然 & BitShares & BTS & Graphene & Cryptomex Team & 2015 \\
\hline (2) & Zcash & Zec & Equicash & Zooko Wilcox & 2016 \\
\hline
\end{tabular}

fonte: CoinGecko, em 2017. elaboração: $\mathrm{O}$ autor.

8 O software da BTC encontra-se disponível no repositório Sourceforge ([s.d.].

9 Algoritmos de hash são aplicações usadas pela comunidade do software livre para garantir a integridade da informação. Esses algoritmos atuam transformando um grande número de dados num pequeno número de informações. Tomando, como exemplo, a distribuição Linux Debian 8.7, ao efetuarmos o download de um arquivo iso para CD-Rom, essa operação de instalação gera no CD-ROM um arquivo contendo um código hash MD5 ou SHA256SUMS, e esse código serve para garantir a segurança e a integridade do registo da informação presentes nas mídias a ser utilizadas, ou seja: 065c90fa72ed51b7ce06ecfdc660b083e7b0c40bbf63fd58559c8bf7df7752da debian-8.7.1-amd64-CD-1.iso; 84e7lfab871dle80518b29d0f3d3f8b2fdd7d2ac2 lb80851fc2cbcdfb8023d9f debian-8.7.1-amd64-CD-2.iso etc. 
Conforme constatação realizada anteriormente, a análise mais atenta dos dados fornecidos pelos sites CoinGecko e MapCoin demonstrou que há uma forte concentração do processo de mineração de moedas virtuais em dois importantes projetos detentores da tecnologia blockchain: a BTC e o ethereum.

modelo de mineração concentrado é o principal desafio a ser enfrentado pelo atual sistema de produção de moedas virtuais, este modelo tem favorecido a tendência de centralização de bitcoins nas mãos de poucos, concentrados nas regiões geográficas mais ricas e grandes mineradores dessas regiões estavam lucrando com a emissão de BTC.10

Existem várias explicações (Prisco, 2017) para esta tendência, a principal é o surgimento de hardwares para mineração especializada, baseados em chips ASICs. " Essa forte concentração é um elemento de risco para o mercado de moedas virtuais, porque não favorece a concorrência. A entrada de novos atores financeiros pode modificar esse cenário de produção.

Procurando reduzir essa tendência de concentração do sistema de mineração de criptomoedas, dois cientistas da computação, os professores da Universidade de Luxemburgo, Alex Biryukov e Dmitry Khovratovich, do grupo de pesquisa CryptoLUX, desenvolveram o algoritmo chamado de Equihash, ${ }^{12}$ utilizado pelo sistema de criptomoedas Zcash, para impedir e resistir ao processo de mineração concentrado executado por hardwares com chips ASICs. Segundo o site CoinGecko, a Zcash assumiu, em abril de 2017, a nona colocação entre os maiores projetos de moedas virtuais no mundo.

\section{A expansão e a concentração geográfica global de operações com moedas virtuais}

A partir de informações fornecidas pela CoinMap, pode-se verificar que, em abril de 2017, a distribuição geográfica de 8961 locais com operações e serviços que utilizam moedas virtuais no mundo (Figura 4), tem ocorrido de uma forma inteiramente diferente e inusitada, principalmente em importantes cidades do mundo. Na geografia, a hierarquia dos lugares centrais sempre esteve atrelada à concentração de serviços e atividades financeiras complexas nas grandes cidades mundiais.

\section{Figura 4 - Concentração geográfica de operações com moedas virtuais}

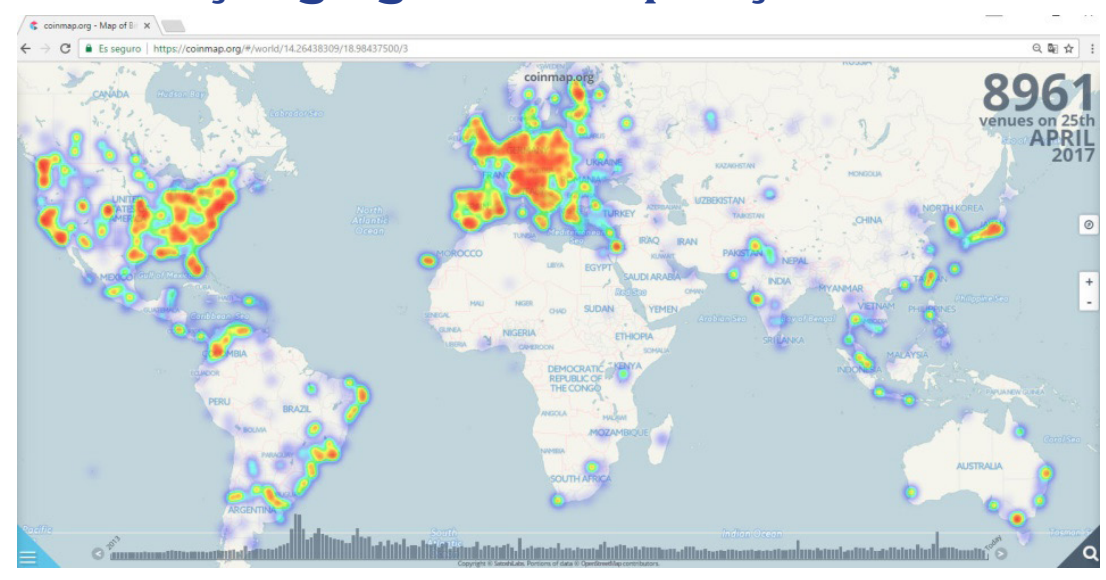

fonte: CoinMap, em 2017.

10 Conferir Luke Dodwell (2017).

11 Conferir no site BitcoinWiki a lista de hardwares ASICs para mineração de Bitcoin (BitcoinWiki, 2017).

12 Sobre o algoritimo de hash Equicash, consultar Biryukov e Khovratovich (2016). 
No período atual, a circulação e os fluxos de moedas virtuais estabeleceram outra hierarquia dos lugares, as cidades mais criativas e propensas a adotar essas inovações produzidas com o uso de moedas virtuais, em suas atividades financeiras e comerciais, estão ocupando o lugar de destaque no uso do dinheiro do ciberespaço, a bitcoin.

Analisando dados e informações fornecidos pela CoinMap, sete cidades no mundo se destacaram na concentração de serviços, em mais de 90 estabelecimentos que utilizam moedas virtuais em suas transações comerciais: São Francisco, Buenos Aires, Nova York, Praga, Arnhem, Vancouver e Londres. Algumas importantes cidades mundiais não conseguiram ainda entrar no ranking das cidades globais com grande concentração de serviços, atividades econômicas e comerciais, que utilizam moedas virtuais em suas transações.

Mas, outras importantes cidades como Buenos Aires, na Argentina, e Atenas, na Grécia, vêm demonstrando um crescimento expressivo e diferenciado na oferta de serviços que utilizam moedas virtuais (Gráfico 3). As enormes dificuldades financeiras que a população dessas cidades sofreu, a redução no volume de moedas circulantes, em diferentes contextos de crises monetárias e, às vezes, o desaparecimento de padrões monetários, talvez seja o fator, que explique o forte crescimento dos usos de moedas virtuais nessas duas cidades.

\section{Gráfico 3 - Concentração geográfica de serviços com moedas virtuais em cidades do mundo}

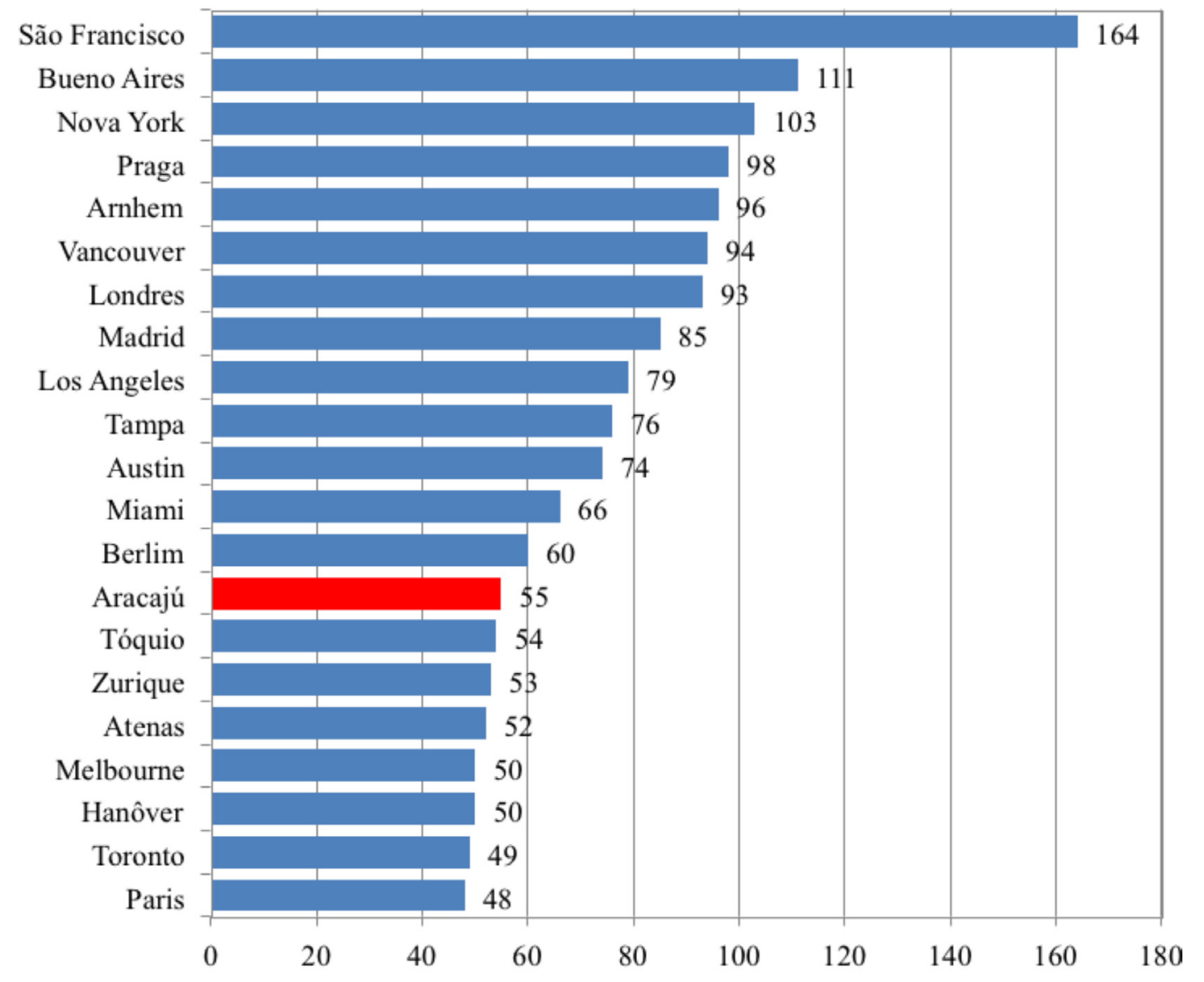

fonte: CoinMap, em 2017. elaboração: $\mathrm{O}$ autor. 
Segundo informações fornecidas pela CoinMap, no Brasil, a cidade mais importante em termos de concentração de serviços em BTC é Aracajú com 55 estabelecimentos, atualmente também chamada de Capital Nacional da Bitcoin, ${ }^{13}$ em segundo lugar, em termos de concentração de serviços e transações comerciais que utilizam a BTC, está São Paulo com 35 estabelecimentos, seguido por Rio de Janeiro com 15 estabelecimentos e Brasília com 14 estabelecimentos (Gráfico 4).

\section{Gráfico 4 - Concentração de serviços que utilizam a BTC em dez cidades brasileiras}

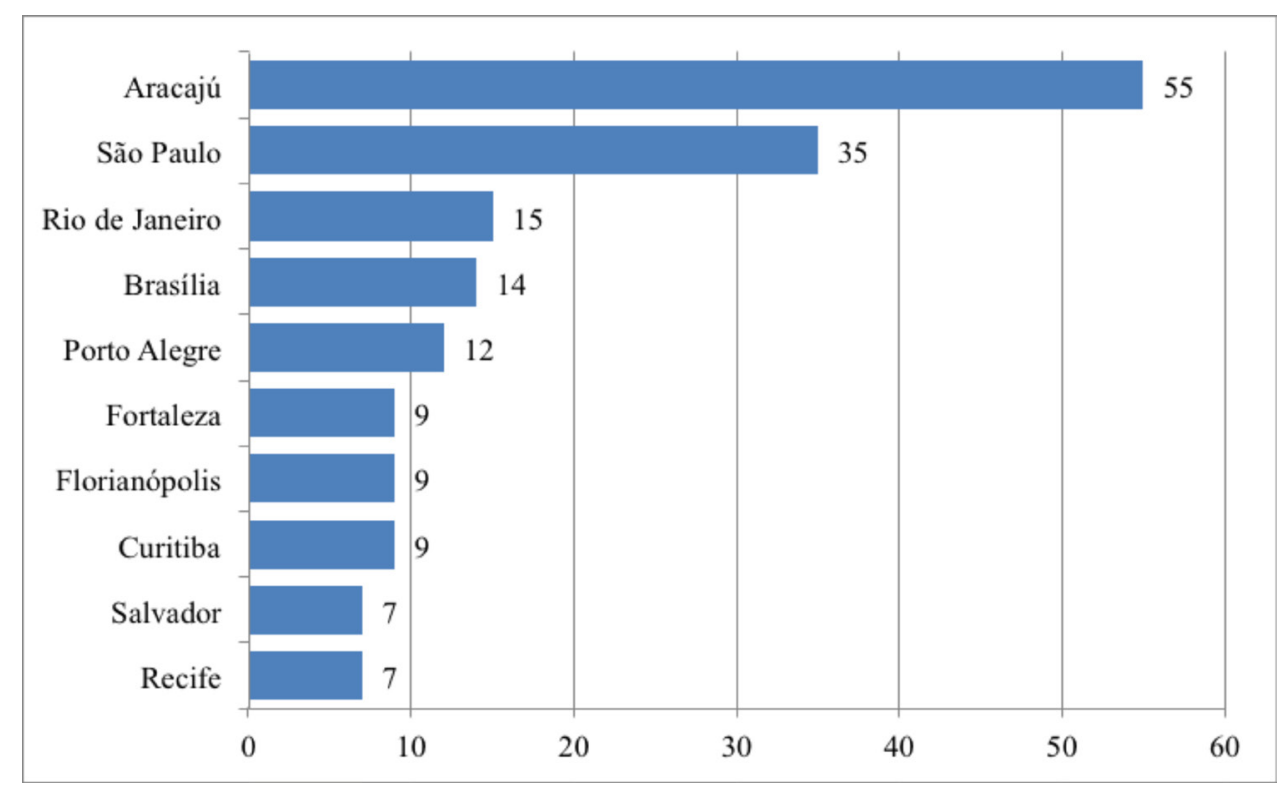

fonte: CoinMap, em 2017. elaboração: $\bigcirc$ autor.

A explicação para o crescimento expressivo do uso de criptomoedas em Aracajú é que essa cidade conquistou esta expertise por uma forte sinergia de fatores criativos: em primeiro lugar, a abertura e a compreensão do empresariado local para os avanços tecnológicos proporcionados com o uso de moedas virtuais em serviços e em atividades comerciais; em segundo lugar, a aceitação de riscos e desafios para promover uma tecnologia em um ambiental comercial profundamente inexperiente, nesse nicho de mercado, e sem tradição no seu uso; em terceiro lugar, a aceitação da ideia de que é necessário aprender a lidar com o novo, principalmente tendo como elemento indutor na formação tecnológica, a Empresa brasileira Rexbit do setor de educação e capacitação, que treinou e ajudou a introduzir os conhecimentos sobre a operacionalização de negócios, que utilizam moedas virtuais como meio de pagamento.

\section{BTC: além do bem e do mal}

Existem algumas "justificativas", na sua grande maioria deturpada, para a não aceitação da BTC e das demais moedas virtuais, como moedas de uso consuetudinário: (a) a vinculação com a cultura hacker e ao anonimato; (b) a imputação que lhe é dada de ser um instrumento

13 Sobre esse assunto, ler a reportagem Aracajú, capital nacional do bitcoin (Vasconcelos, 2017, p. 32). 
político de sonegação e evasão fiscal global; (c), a sua não submissão à regulação jurídica dos Estados nacionais e dos bancos; (d), a de ser uma tecnologia acusada de promover o mall ${ }^{14} \mathrm{e}$ serviços sem segurança contratual.

Segundo esse discurso, a BTC e demais moedas virtuais foram concebidas para inviabilizar os mecanismos de regulação dos bancos centrais e foi criado para frear a capacidade dos Estados nacionais de cobrar impostos e fiscalizar as transações financeiras de seus cidadãos. ${ }^{15}$

A propagação ideologizada dessas "assertivas" conduziu a uma oposição contra a BTC, desencadeada por vários países e agências de segurança nacional.

Sem muita evidência, os governos em muitos países acusam a BTC de ser usada para encorajar operações ilegais, como compras de armas, lavagem de dinheiro, terrorismo e uso de drogas. Segundo as autoridades que combatem crimes na internet, as plataformas que operam com moedas virtuais, como por exemplo: Tor, Deep Web e Silk Road, utilizam a BTC para promover ações criminosas em várias regiões do mundo. Recentemente, governos de 10 países proibiram o uso de BTC são eles: Bangladesh, Bolívia, China, Equador, Islândia, Índia, Rússia, Suécia, Tailândia e Vietnã.

Os argumentos utilizados por esses governos, para adoção de medidas de proibição territorial (Figura 5) do uso de criptomoedas, foram: (a) a BTC opera em um ambiente de elevado grau de anonimato global; (b) a BTC pode se tornar uma ferramenta para diversos tipos de crimes; (c) a BTC não pode ser controlado por Estados e nem regulado por autoridades bancárias; e (d) a BTC pode provocar evasão e lavagem de dinheiro.

\section{Figura 5 - Mapa global do cenário jurídico do BTC em 2017}

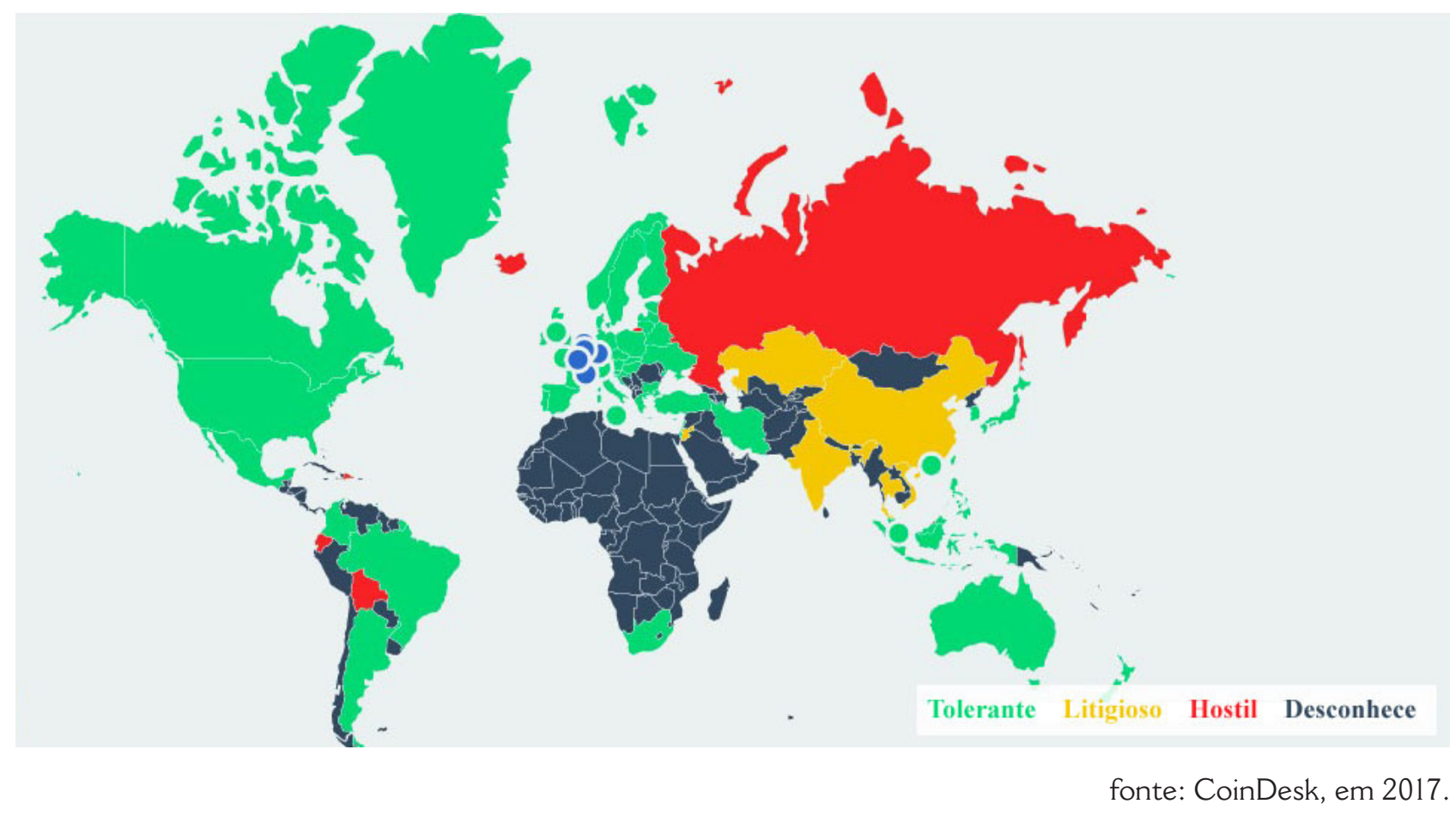

14 Conferir Paul Krugman (2013).

15 Ler a crítica radical Why I want Bitcoin to die in a fire (Stross, 2013). 
Mesmo sendo proibido na China continental, a BTC é fortemente usada na China Insular (Hong Kong e Taiwan), as operações de moedas virtuais podem ser identificadas e visualizadas com facilidade, nos sites da CoinMap e no FiatLeak. Segundo a Bloomberg, ${ }^{16}$ a China é responsável por 90\% dos negócios em BTC e 70\% das operações de mineração de moedas virtuais no mundo. Alguns operadores de BTC, em Hong Kong, chegam a ter, em sua carteira de bitcoins, mais de um milhão de usuários em todo o mundo, como é o caso ANX International ([s.d.]).

Diferentemente do que ocorre em outros países, que implementam medidas de restrição ao uso de moedas virtuais, na Holanda, esse uso já se encontra bastante difundido em importantes cidades como Amsterdam, Arnhem e na zona portuária próxima de Roterdam; na Alemanha, a circulação de BTC ocorre sem restrições em várias cidades alemães como Berlim, Hanôver e Hamburgo.

Nos EUA, segundo o no relatório Application of FinCEN's Regulations to Virtual Currency Mining Operations, elaborado pela Rede de Execução de Crimes Financeiros, ou Financial Crimes Enforcement Network (FinCEN), em 30 de janeiro de 2014, o usuário de moedas virtuais:

[... é é considerado livre para usar a moeda virtual extraída ou seu equivalente para os seus próprios propósitos, como comprar bens e serviços reais ou virtuais para uso próprio do usuário. [...] E, claro, se a [Companhia] se envolver em qualquer outra atividade que constitua aceitação e transmissão de qualquer moeda de curso legal ou moeda virtual, poderá realizar atividades de transmissão de dinheiro que estariam sujeitas aos requisitos da lei de sigilo bancário ou Bank Secrecy Act (BSA) (Fincen, 2014).

Assim, o uso de moedas virtuais não é considerado ilegal nos EUA, porque é preservado o princípio da escolha do cidadão e atualmente, esse país é o que mais utiliza moedas virtuais para transações e operações pessoais; por isso, o seu uso cresceu em importantes cidades estadunidenses como: São Francisco, Nova York, Los Angeles, Tampa, Austin e Miami (Fincen, 2014).

\section{Da chegada do dinheiro da globalização ao dinheiro do ciberespaço}

Em 1999, ao escrever sobre a chegada do dinheiro da globalização, Milton Santos previu que este seria originado das técnicas da informação, que por sua vez proporcionaria a fluidez efetiva a serviço de capitais globalizados, de tal modo que o dinheiro apareceria como dinheiro global fluido, impondo caminhos às nações. Segundo ainda ele, se o dinheiro que comanda é dinheiro global, o território ainda resistirá: " $\bigcirc$ equivalente geral torna-se afinal o equivalente realmente universal. Mas esse dinheiro não é sustentado por operações da ordem da infraestrutura" (Santos, 1999, p. 10; 2000).

Em junho 2014, os pesquisadores David Malone e Karl O’Dwyer demonstraram por meio de cálculos estatísticos, apresentados na pesquisa Bitcoin Mining and its Energy Footprint (2014), que a rede de computadores P2P utilizada para minerar e produzir criptomoedas em

16 Conferir o vídeo e o artigo de Chen e Nakamura (2017). 
escala planetária, consumia proporcionalmente o equivalente a energia elétrica gasta pela Irlanda. Nesse sentido, a nova forma de produzir dinheiro, no século XXI, tende a ser "sustentada" sim, por operações da ordem da infraestrutura para a mineração de moedas virtuais.

Assim como a internet global, a produção e o uso de moedas virtuais necessitam também de uma complexa infraestrutura crítica de redes tecnológicas, de energia elétrica e de tecnologias da informação e comunicação. Essa infraestrutura nem é sempre visível aos olhos de seus usuários. Nesse sentido, a produção de BTC, uma moeda do ciberespaço, por ser uma tecnologia P2P, requer uma grande quantidade de consumo de energia e processamento de dados para processar as diferentes formas de criptomoedas.

$\bigcirc$ discurso ideologizado utiliza-se, muitas vezes, da "assertiva" que as moedas virtuais consomem muita energia, mas é preciso refletir também sobre: (a) os custos existentes para emissão de moedas "reais" em vários países, em suas diferentes formas (papel, metal, plástico); (b) a logística concreta e virtual de armazenamento do dinheiro "real" nos bancos; e (c) os custos com seguro, segurança e transporte de valores, que também consomem muita energia.

Contrariando essa tendência de insustentabilidade da emissão de moedas virtuais, existem vários projetos em desenvolvimento para a mineração de criptomoedas que fazem uso de energias alternativas: solar e eólica. ${ }^{17}$

\section{Considerações finais}

Formado por 30 dos maiores bancos do mundo, o Consórcio Global R3CEV, em 2015, havia decidido se articular para produzir serviços financeiros globais baseados na tecnologia da blockchain. Em 2017, esse consórcio oficializou sua desistência ${ }^{18}$ no desenvolvimento de tecnologia de contabilidade distribuída inspirada na blockchain, isso depois de ter gasto mais de 59 milhões de dólares em pesquisa e desenvolvimento com a tecnologia da blockchain.

Isso pode indicar que o capitalismo financeiro não conseguiu ainda se adaptar e apropriar dos elementos de inovação gerados pelas tecnologias de inteligência computacional, baseadas nos algoritmos da blockchain, ou considera que seus lucros poderiam não ser tão vantajosos como são atualmente no cassino das moedas podres e deslastreadas.

Mesmo assim, continua crescendo a aceitação da BTC por importantes empresas de tecnologia (Dell, Google, Microsoft, LibreOffice, WordPress.com, Itunes, Mega.co.nz etc. $)^{19}$ e por organizações da sociedade civil que defendem a liberdade de expressão, a neutralidade na rede, a liberdade de organização dos movimentos sociais, o direito à privacidade, o uso de softwares livres (Pirate Party, Electronic Frontier Foundation, Wikileaks) e o uso de uma moeda virtual que tenha uso social com menos riscos e lucros bancários especulativos.

Algo semelhante também começa a acontecer com as organizações que fazem trabalhos de ajuda humanitária e caridade (Wikimedia Foundation, Greenpeace, United Way Worldwide, Save the Children), que atualmente estão aceitando a BTC como instrumento de financiamento de suas ações.

17 Conferir artigo de Holmes (2015).

18 Sobre esse assunto, conferir o artigo de William Suberg (2017).

19 Conferir a reportagem Who Accepts Bitcoins? (Chokun, 2017). 


\section{Referências}

ANX International. Disponível em: https://anxintl.com/aboutus/. Acesso em: 12 maio 2017.

BANKY Moon. Bitcoin solving an African problem, 12 out. 2016. Disponível em: http://bankymoon.co.za/2016/10/12/bitcoin-solving-an-african-problem/. Acesso em: 20 jul. 2017.

BIRYUKOV, A.; KHOVRATOVICH, D. Equihash: asymmetric proof-of-work based on the Generalized Birthday problem. NETWORK AND DISTRIBUTED SYSTEM SECURITY SYMPOSIUM (NDSS), 2016, San Diego, CA. Proceedings... San Diego, CA, 2016. Disponível em: http://orbilu.uni.lu/bitstream/10993/22277/2/946.pdf. Acesso em: 12 maio 2017.

BITCOINWIKI. Lista de hardwares ASICs para mineração de bitcoin, 2017. Disponível em: https:// en.bitcoin.it/wiki/List_of_Bitcoin_mining_ASICs. Acesso em: 20 jun. 2017.

COINCAP. Disponível em: http://coincap.io/. Acesso em: 17 maio 2017.

COINDESK. Disponível em: http://www.coindesk.com/bitcoin-legal-map/. Acesso em: 17 maio 2017.

COINGECKO. Disponível em: https://www.coingecko.com/. Acesso em: 27 jun. 2017.

COINMAP. Disponível em: https://coinmap.org/. Acesso em: 12 maio 2017.

CHEN, L. Y; NAKAMURA, Y. After 4,400\% Surge, Bitcoin's Fate Hinges on Huge Chinese Miners. Bloomberg, jul. 2017. Disponível em: https://www.bloomberg.com/news/articles/2016-07-06/after-4-400-surge-bitcoin-s-fate-hinges-on-huge-chinese-miners. Acesso em: 20 jun. 2017.

CHOKUN, J. Who Accepts Bitcoins? 99 Bitcoins, 28 maio 2017. Disponível em: https://99bitcoins. com/who-accepts-bitcoins-payment-companies-stores-take-bitcoins/. Acesso em: 20 jun. 2017.

DODWELL, L. Is Mining Bitcoin Still Profitable?. Crush the street, 14 fev. 2017. Disponível em: https://crushthestreet.com/articles/digital-currencies/mining-bitcoin-profitable. Acesso em: 20 jun. 2017.

IGF. INTERNET GOVERNANCE FORUM. Blockchain Technologies IGF 2016 - Day 3 - Room 8 - DC on Blockchain Technologies. Jalisco, MX, dez. 2016. Disponível em: https://www. intgovforum.org/multilingual/content/igf-2016-day-3-room-8-dc-on-blockchain-technologies. Acesso em: 25 jun. 2017.

INTERNET GOVERNANCE FORUM. Blockchain and Beyond: Flash Help!. IGF 2015 Day 4 - WK 3 - Dynamic Coalition on Blockchain Technologies, 13 nov. 2015. Disponível em: https://www.youtube.com/watch?v=QXNnG9VGwwA. Acesso em: 20 jun. 2017.

FIATLEAK. Disponível em: http://fiatleak.com/. Acesso em: 20 jul. 2017.

FINCEN. FINANCIAL CRIMES ENFORCEMENT NETWORK. Application of FinCEN's Regulations to Virtual Currency Mining Operations, 30 jan. 2014. Disponível em: https://www.fincen.gov/sites/default/files/administrative_ruling/FIN-2014-R001. pdf. Acesso em: 10 maio 2017. 
HOLMES, B. Bitcoin and Sustainable Mining. Brave NewCoin, 16 jul. 2015. Disponível em: https://bravenewcoin.com/news/bitcoin-and-sustainable-mining/. Acesso em: 10 maio 2017.

KRUGMAN, P. Bitcoin Is Evil. The New York Times, New York, 28 dec. 2013. The Opinion Pages. Disponível em: http://krugman.blogs.nytimes.com/2013/12/28/bitcoin-is-evil/. Acesso em: 15 jun. 2017.

MALONE, D.; O’DWYER, K. J. Bitcoin Mining and Its Energy Footprint. IRISH SIGNALS E SYSTEMS CONFERENCE (ISSC) AND CHINA-IRELAND INTERNATIONAL CONFERENCE (CIIT), 2014, Limerick, IRL, Proceedings... Limerick, IRL, 2014. p. 280-285. Disponível em: https://karlodwyer.github.io/publications/pdf/bitcoin_KJOD_2014.pdf. Acesso em: 12 maio 2017.

MAP of Coins. Disponível em: http://mapcoins.com/. Acesso em: 12 maio 2017.

NAKAMOTO, S. Bitcoin: A Peer-to-Peer Electronic Cash System. In: Bitcoin Org, 2008. Disponível em: https://bitcoin.org/bitcoin.pdf. Acesso em: 12 maio 2017.

PIRES, H. F. The Globalization of Cryptocurrency: The end of banks and financial intermediation? In: ANNUAL MEETING OF THE AMERICAN ASSOCIATION OF GEOGRAPHERS, 2017, Boston. Annual Meeting Program, Boston, 2017. p. 389-390.

- Capitalismo gestor de dinheiro e desregulação financeira: o endividamento dos EUA e das economias centrais. In: PACHECO, S. M. M.; MACHADO, M. S. (Org.). Globalização, políticas públicas e reestruturação territorial. Rio de Janeiro: 7Letras, 2012. p. 109-143. v. 1. Disponível em: http://www.ub.edu/geocrit/b3w-963.htm. Acesso em: 12 maio 2017.

- Redes sociais colaborativas e geografia em rede: as novas formas de apropriação do conhecimento social no século XXI. Revista Terra Livre, São Paulo, ano 26, v. 1, n. 34, p. 17-36, jan./jun. 2010. Disponível em: http://www.agb.org.br/publicacoes/index.php/terralivre/article/ view/308. Acesso em: 12 maio 2017.

PRISCO, G. How the Equihash Algorithm Could Democratize Zcash Mining. Bitcoin Magazine, 16 fev. 2017. Disponivel em: https://bitcoinmagazine.com/articles/how-equihash-algorithm-could-democratize-zcash-mining/. Acesso em: 12 maio 2017.

SANTOS, M. Por uma outra globalização: do pensamento único à consciência universal. Rio de Janeiro: Record, 2000.

. O dinheiro e o território. GEOgraphia, Niterói, RJ, v. 1, n. 1, 1999. Disponível em: http:// www.uff.br/geographia/ojs/index.php/geographia/article/download/2/2-8-1-PB.pdf. Acesso em: 12 maio 2017.

SAYAD, J. Dinheiro, dinheiro: inflação, desemprego, crises financeiras e bancos. São Paulo: Portfolio Peguin, 2015.

SCHED. Dynamic Coalition on Blockchain Technology. IGF 2015 de João Pessoa, BR, 13 nov. 2015. Disponível em: https://igf2015.sched.com/event/4c1G/dynamic-coalition-on-blockchain-technologiesBitcoin. Acesso em: 25 jul. 2017. 
SCHWAB, K. The Fourth Industrial Revolution. Geneva: World Economic Forum, 2016.

SOURCEFORGE. Disponível em: https://sourceforge.net/projects/bitcoin/. Acesso em: 10 jun. 2017.

STROSS, C. Why I want Bitcoin to die in a fire. Charlie's Diary Blog, dec. 2013. Disponível em: http://www.antipope.org/charlie/blog-static/2013/12/why-i-want-bitcoin-to-die-in-a.html. Acesso em: 10 maio 2017.

SUBERG, W. We Don't Need Blockchain: R3 Consortium After \$59 Million Research. The cointelegraph, 22 fev. 2017. Disponível em: https://cointelegraph.com/news/we-dont-need-blockchain-r3-consortium-after-59-million-research. Acesso em: 10 maio 2017.

VASCONCELOS, A. (Ed.). Guia mundo em foco especial atualidades: Bitcoin - o dinheiro do futuro?. São Paulo: On line, 2017.

YELOWITZ, A.; WILSON, M. Characteristics of Bitcoin users: an analysis of Google search data. Applied Economics Letters, v. 22, n. 13, p. 1030-1036, 2015. Disponível em: http://dx.doi. org/10.1080/13504851.2014.995359. Acesso em: 12 maio 2017. 